\title{
ATG in allogeneic stem cell transplantation: standard of care in 2017? Point
}

Andrea Bacigalupo

Fondazione Policlinico Universitario A. Gemelli, Università Cattolica del Sacro Cuore, Rome, Italy

This article has a companion Counterpoint by Kekre and Antin.

Graft-versus-host disease (GVHD), both in its acute and chronic form, is associated with considerable morbidity and mortality. ${ }^{1}$ In the past decade, acute GVHD (aGVHD) has been reduced as the result of improved prophylaxis. ${ }^{2}$ On the contrary, chronic GVHD (cGVHD) has shown a significantly increased rate, due to the widespread use of peripheral blood (PB) cells as a stem cell source, older patient age, and more alternative donor transplants, all of which are known risk factors for cGVHD. ${ }^{3}$

\section{Anti-thymocyte globulin (ATG): randomized trials}

ATG has been used with different donor types: HLA-identical siblings (SIB), unrelated donors (UDs), and cord blood (CB) haploidentical-related donors. Two randomized trials before the cyclosporine era, had shown the protective effect of ATG against aGVHD., 4 Three more recent prospective trials have been conducted in Europe, randomizing patients to receive, or not to receive ATG, in the conditioning regimen. ${ }^{6-10}$ All 3 studies compared a conventional cyclosporine-methotrexate GVHD prophylaxis, with or without the addition of ATG, and are summarized in Table 1. The first study, published in $2001^{6}$ and updated in $2006^{7}$ was conducted by the GITMO, with rabbit ATG (thymoglobuline; Sanofi-Genzyme, Saint-Germain-en-Laye, France): all patients received grafts from UDs, following a myeloablative (MA) regimen and bone marrow $(B M)$ was the stem cell source. There was a significant reduction of aGVHD and cGVHD, and survival was comparable. In the 2006 update, a strong protection against chronic lung dysfunction and improved quality of life was evident for patients receiving ATG. ${ }^{7}$ The second study was conducted 10 years later by the German Cooperative Group, published in $2009^{8}$ and updated in 2011 9 : the ATG brand was rabbit ATG (Fresenius, Hamburg, Germany); the donors were UD, the conditioning regimen was $M A$, and the stem cell source was mainly granulocyte colony-stimulating factor-mobilized PB; aGVHD and cGVHD were reduced and survival was unchanged, but survival free of immunosuppressive therapy was $52 \%$ for ATG patients vs $17 \%$ for non-ATG patients. ${ }^{9}$ The third study is noteworthy because it was conducted in patients receiving SIB PB grafts, following an MA regimen. ${ }^{10}$ The cumulative incidence of cGVHD was $32 \%$ in the ATG group vs $68 \%$ in the non-ATG group $(P<.0001)$, and the GVHD relapse-free survival was $37 \%$ vs $17 \%(P=.005)$. Overall survival and disease-free survival were unchanged.

The setting of these 3 trials is therefore quite different: different transplant era, different matching standards for UD grafts, different stem cell sources, different donors, and different ATG brands. Despite these significant differences, results in terms of aGVHD and cGVHD are quite reproducible in the different trials; in particular, the protection against extensive cGVHD is almost identical for patients receiving ATG, and is shown in Figure 1. Table 1 summarizes the results of the 3 randomized trials: for patients not receiving ATG, the average increased risk of aGVHD grades II-IV was 1.47-fold, for grades III-IV it was twofold, for cGVHD it was 1.83-fold, and for extensive cGVHD it was 3.43-fold (from 11\% to $37 \%$ ); in non-ATG patients, the probability of NRM was increased, ${ }^{1}$ the rate of relapse was decreased (0.84-fold), and survival was comparable (1.04-fold).The great difference in extensive cGVHD, averaging almost a 3.5-fold increased risk for patients not receiving ATG, does not translate in these 3 randomized studies, in an increased risk of relapse nor in decreased NRM and improved overall survival (Table 1). However, 1 study with long follow up, showed a significant reduction of chronic pulmonary dysfunction in patients receiving ATG, and a trend toward lower late NRM. ${ }^{7}$ Thus, 3 randomized studies in different settings have come up with very similar results: protection from aGVHD and cGVHD, and comparable survival.

\section{ATG and leukemia relapse}

The crucial question is whether protection against GVHD translates in increased relapse, and whether ATG should not be given to patients with advanced disease. The 3 randomized studies ${ }^{6-10}$ have not shown significant increased risk of relapse, and this is true also for the Canadian study: a meta-analysis on a large number of patients would disprove increased relapse in patients receiving ATG in the conditioning regimen. ${ }^{11}$ Therefore, in keeping with the results of the randomized trials, ATG is used in my 


\begin{tabular}{|c|c|c|c|c|c|c|c|c|c|c|}
\hline & \multicolumn{2}{|c|}{ GITMO $^{6,7}$} & \multicolumn{2}{|c|}{ Finke $^{8,9}$} & \multicolumn{2}{|c|}{ Kröger $^{10}$} & \multicolumn{2}{|c|}{ Total } & \multirow[b]{2}{*}{$\mathbf{R R}$} & \multirow[b]{2}{*}{$\boldsymbol{P}$} \\
\hline & ATG & nOATG & ATG & nOATG & ATG & nOATG & ATG & nOATG & & \\
\hline Patients, $\mathrm{n}$ & 56 & 53 & 103 & 98 & 83 & 72 & 242 & 223 & - & - \\
\hline aGVHD ॥-IV, \% & $50 \%$ & $70 \%$ & $33 \%$ & $51 \%$ & $11 \%$ & $18 \%$ & $31 \%$ & $46 \%$ & 1.47 & .001 \\
\hline aGVHD III-IV, \% & $23 \%$ & $43 \%$ & $11 \%$ & $24 \%$ & $2 \%$ & $8 \%$ & $12 \%$ & $25 \%$ & 2.08 & .0003 \\
\hline cGVHD, \% & $37 \%$ & $60 \%$ & $26 \%$ & $50 \%$ & $22 \%$ & $46 \%$ & $28 \%$ & $52 \%$ & 1.83 & .00001 \\
\hline ext cGVHD, \% & $15 \%$ & $41 \%$ & $12 \%$ & $45 \%$ & $5 \%$ & $24 \%$ & $11 \%$ & $37 \%$ & 3.43 & .00001 \\
\hline NRM, \% & $39 \%$ & $47 \%$ & $19 \%$ & $33 \%$ & $14 \%$ & $12 \%$ & $24 \%$ & $31 \%$ & 1.27 & .1 \\
\hline Relapse, \% & $23 \%$ & $21 \%$ & $33 \%$ & $28 \%$ & $32 \%$ & $25 \%$ & $29 \%$ & $25 \%$ & 0.84 & .2 \\
\hline Survival, \% & $55 \%$ & $56 \%$ & $55 \%$ & $43 \%$ & $74 \%$ & $77 \%$ & $61 \%$ & $59 \%$ & 1.04 & .6 \\
\hline
\end{tabular}

ext, extensive; GITMO, Italian Cooperative Transplant Group; NRM, non-relapse mortality; RR, relative risk of patients not receiving ATG as compared with ATG.

unit and largely in Europe, not only for patients with early disease, but also for patients with advanced leukemia.

\section{Dose of ATG}

Several issues remain to be solved in the use of ATG in allogeneic transplantation: the optimal dose has not been identified precisely, also because it differs in the two currently available brands (thymoglobulin and ATG Fresenius [ATG-F]), and may depend on the donor type, as recently reviewed. ${ }^{12}$ For SIB transplants, the recent randomized study would support the use of ATG-F at the dose of 30 $\mathrm{mg} / \mathrm{kg}^{10}$ whereas retrospective studies would indicate a dose of thymoglobuline ranging from 2.5 to $5 \mathrm{mg} / \mathrm{kg} .{ }^{13}$ For matched UD transplants, the dose of ATG-F would be $60 \mathrm{mg} / \mathrm{kg}$ and the dose of thymoglobuline 4.5 to $7.5 \mathrm{mg} / \mathrm{kg} .{ }^{11-14} \mathrm{We}$ have randomized thymoglobuline 7.5 vs $10 \mathrm{mg} / \mathrm{kg}$ for UD grafts, and have additional protection with the higher dose against severe aGVHD and extensive cGVHD, ${ }^{15}$ but with no impact of NRM, relapse, and survival.

\section{Timing of ATG and blood levels}

The interval between ATG and the infusion is the other significant issue: the closer to transplant, the higher the levels of circulating ATG, and the latter are crucial for GVHD protection. ${ }^{16,17}$ Usually, ATG is administered on days -3-2-1 before transplant.

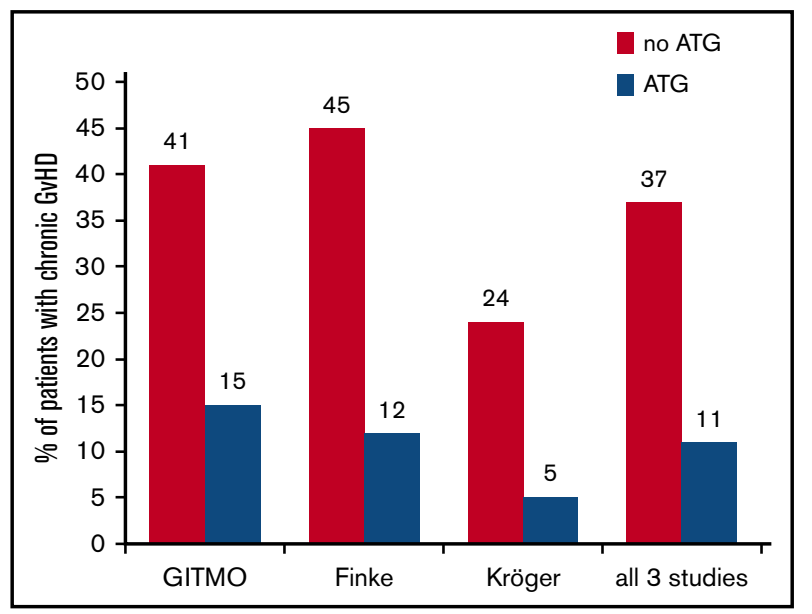

Figure 1. Percentage of patients with severe cGVHD in 3 randomized trials. (GITMO, ${ }^{6,7}$ Finke, ${ }^{8,9}$ Kröger, $^{10}$ and combined data [total] are shown).

\section{Early ATG toxicity}

ATG must be given in experienced centers, because early toxicity such as allergic reactions and also increased bilirubin levels can require prompt medical intervention. Delayed side effects, such as an increased risk of Epstein-Barr virus reactivation, must also be taken in to account, and may call for the preventive use of rituximab. ${ }^{18}$

\section{ATG and CB transplants (CBT)}

One important issue is whether CBT should be performed with or without the use of ATG: in a recent Registry-based study, NRM was significantly increased in patients receiving a CBT with ATG, as compared with CBT without ATG (RR, 1.63; $P=.03$ ), and leukemiafree survival was significantly decreased (RR, 1.29; $P=.02) .^{19}$ This is probably due to an increased risk of infectious complications with ATG, in a setting already exposed to this problem due to slow engraftment and prolonged immune deficiency.

In conclusion, in the era of monoclonal humanized antibodies, it seems hard to believe that we are still using ATG, a crude product of immunization across species, more than half a century after its introduction in the BM transplant arena by Mathe and Schwarzenberg. ${ }^{20}$ The fact remains that ATG has been shown to protect patients from aGVHD and cGVHD in prospective randomized trials, without reducing survival. In other words, the probability of a patient being alive 5 years posttransplant is the same, whether he/she receives ATG or not; the difference lies in cGVHD, quality of life, and immunosuppressive therapy. For this reason, ATG has been used in Europe for all patients receiving alternative donor grafts; following the randomized trial in SIBs, ATG is increasingly used in SIB transplants, when $\mathrm{PB}$ is the stem cells source. Based on the lack of effective measures to treat $\mathrm{cGVHD},{ }^{21}$ the author strongly agrees with the current use of ATG in Europe for all UD transplants, as well as for SIB grafts when PB is the stem cells source. In addition, the author is aware that this is not the case in the United States, where based on a retrospective study ${ }^{22}$ and now also on a recent prospective study, ${ }^{23}$ ATG is thought to increase the risk of relapse. Retrospective studies are always questionable because of a possible selection bias. The recent prospective randomized trial ${ }^{23}$ confirms the protection of ATG on aGVHD and cGVHD but has failed to show a benefit, due to a dramatic survival difference in a subset of patients prepared with cyclophosphamide total body irradiation (a minority), whereas no survival difference was seen in patients receiving busulfan-based regimens. For these reasons, 
most transplants in the United States still perform UD transplants without ATG, and this will expose patients to a significant risk of aGVHD and especially cGVHD, with a decrease in the quality of life. Of the 4 randomized ATG trials, ${ }^{7,9,10}$ all 4 have shown protection against cGVHD, and 3 of them without a detrimental effect on survival: the bulk of the data strongly suggests that we should use ATG for all allogeneic transplants at high risk of GVHD.

\section{Acknowledgment}

This work was supported by the Fondazione Ricerca Trapianto Midollo Osseo Research Foundation Bone Marrow Transplantation, Genoa, Italy.

\section{Authorship}

Contribution: A.B. wrote this manuscript.

Conflict-of-interest disclosure: A.B. is on the Speaker's Bureau for Sanofi, Merck, Adienne, Therakos, Miltenyi, and Pierre Fabre.

Correspondence: Andrea Bacigalupo, Fondazione Policlinico Universitario A. Gemelli, Università Cattolica del Sacro Cuore, Largo A. Gemelli 1, 00168 Rome, Italy; e-mail: apbacigalupo@yahoo.com.

\section{References}

1. Gratwohl A, Brand R, Apperley J, et al; Chronic Leukemia Working Party of the European Group for Blood and Marrow Transplantation (CLWP-EBMT). Graft-versus-host disease and outcome in HLA-identical sibling transplantations for chronic myeloid leukemia. Blood. 2002;100(12):3877-3886.

2. Gooley TA, Chien JW, Pergam SA, et al. Reduced mortality after allogeneic hematopoietic stem cell transplantation. N Engl J Med. 2010;363(22):2091-2101.

3. Arai S, Arora M, Wang T, et al; Graft-vs-Host Disease Working Committee of the CIBMTR. Increasing incidence of chronic graft-versus-host disease in allogeneic transplantation: a report from the Center for International Blood and Marrow Transplant Research. Biol Blood Marrow Transplant. 2015; 21(2):266-274.

4. Ramsay NK, Kersey JH, Robison LL, et al. A randomized study of the prevention of acute graft-versus-host disease. $N$ Engl $J$ Med. 1982;306(7):392-397.

5. Weiden PL, Doney K, Storb R, Thomas ED. Antihuman thymocyte globulin for prophylaxis of graft-versus-host disease. A randomized trial in patients with leukemia treated with HLA-identical sibling marrow grafts. Transplantation. 1979;27(4):227-230.

6. Bacigalupo A, Lamparelli T, Bruzzi P, et al. Antithymocyte globulin for graft-versus-host disease prophylaxis in transplants from unrelated donors: 2 randomized studies from Gruppo Italiano Trapianti Midollo Osseo (GITMO). Blood. 2001; 98(10):2942-2947.

7. Bacigalupo A, Lamparelli T, Barisione G, et al; Gruppo Italiano Trapianti Midollo Osseo (GITMO). Thymoglobulin prevents chronic graft-versus-host disease, chronic lung dysfunction, and late transplant-related mortality: long-term follow-up of a randomized trial in patients undergoing unrelated donor transplantation. Biol Blood Marrow Transplant. 2006;12(5): 560-565.
8. Finke J, Bethge WA, Schmoor C, et al; ATG-Fresenius Trial Group. Standard graft-versus-host disease prophylaxis with or without anti-T-cell globulin in haematopoietic cell transplantation from matched unrelated donors: a randomised, open-label, multicentre phase 3 trial. Lancet Oncol. 2009; 10(9):855-864.

9. Socie G, Schmoor C, Bethge WA, et al. Chronic graft-versushost disease: long-term results from a randomized trial on graft-versus-host disease prophylaxis with or without anti-T-cell globulin ATG Fresenius. Blood. 2011;117(23):6375-6382.

10. Kröger N, Solano C, Wolschke C, et al. Antilymphocyte globulin for prevention of chronic graft-versus-host disease. N Engl J Med. 2016;374(1):43-53.

11. Kumar A, Mhaskar AR, Reljic T, et al. Antithymocyte globulin for acute-graft-versus-host-disease prophylaxis in patients undergoing allogeneic hematopoietic cell transplantation: a systematic review. Leukemia. 2012;26(4):582-588.

12. Baron F, Mohty M, Blaise D, et al. Anti-thymocyte globulin as graft-versus-host disease prevention in the setting of allogeneic peripheral blood stem cell transplantation: a review from the Acute Leukemia Working Party of the European Society for Blood and Marrow Transplantation. Haematologica. 2017;102(2):224-234.

13. Crocchiolo $R$, Esterni $B$, Castagna $L$, et al. Two days of antithymocyte globulin are associated with a reduced incidence of acute and chronic graft-versus-host disease in reduced-intensity conditioning transplantation for hematologic diseases. Cancer. 2013;119(5):986-992.

14. Bashir Q, Munsell MF, Giralt S, et al. Randomized phase II trial comparing two dose levels of thymoglobulin in patients undergoing unrelated donor hematopoietic cell transplantation. Leuk Lymphoma. 2012;53(5):915-919.

15. Bacigalupo A, Lamparelli T, Milone G, et al; Gruppo Italiano Trapianto Midollo Osseo (GITMO). Pre-emptive treatment of acute GVHD: a randomized multicenter trial of rabbit antithymocyte globulin, given on day +7 after alternative donor transplants. Bone Marrow Transplant. 2010;45(2):385-391.

16. Remberger $M$, Sundberg B. Low serum levels of total rabbit$\lg \mathrm{G}$ is associated with acute graft-versus-host disease after unrelated donor hematopoietic stem cell transplantation: results from a prospective study. Biol Blood Marrow Transplant. 2009;15(8):996-999.

17. Remberger $M$, Sundberg B. Rabbit-immunoglobulin $G$ levels in patients receiving thymoglobulin as part of conditioning before unrelated donor stem cell transplantation. Haematologica. 2005;90(7):931-938.

18. Dominietto $A$, Tedone $E$, Soracco $M$, et al. In vivo B-cell depletion with rituximab for alternative donor hemopoietic SCT. Bone Marrow Transplant. 2012;47(1):101-106.

19. Ruggeri A, Labopin M, Sanz G, et al; Eurocord, Cord Blood Committee of Cellular Therapy and Immunobiology working party-EBMT; ALWP-EBMT study. Comparison of outcomes after unrelated cord blood and unmanipulated haploidentical stem cell transplantation in adults with acute leukemia. Leukemia. 2015;29(9):1891-1900. 
20. Mathe G, Schwarzenberg L. Bone marrow transplantation (1958-1978) : conditioning and graft-versus-host disease, indications in aplasias and leukemias. Pathol Biol (Paris). $1979 ; 27(6): 337-343$.

21. Palmer J, Chai X, Martin PJ, et al. Failure-free survival in a prospective cohort of patients with chronic graft-versus-host disease. Haematologica. 2015;100(5):690-695.

22. Soiffer RJ, Lerademacher J, Ho V, et al. Impact of immune modulation with anti-T-cell antibodies on the outcome of reduced-intensity allogeneic hematopoietic stem cell transplantation for hematologic malignancies. Blood. 2011; 117(25):6963-6970.

23. Soiffer RJ, Kim HT, McGuirk J, et al.A prospective randomized double blind phase 3 clinical trial of anti-T lymphocyte globulin (ATLG) to assess the impact of chronic graft-versus-host disease (cGVHD) free survival in patients undergoing HLA matched unrelated myeloablative hematopoietic cell transplantation (HCT). Blood. 2016;128(22):505.

DOI 10.1182/bloodadvances.2016001560

(C) 2017 by The American Society of Hematology 